\title{
ArcheoSciences
}

Revue d'archéométrie

\section{Modéliser la gestion des grandes lames en silex de la fin du Néolithique à partir de la quantification des traces d'utilisation}

Modelling the management of late Neolithic large flint blades through use-wear quantification

\section{Jimmy Linton}

\section{OpenEdition}

Journals

\section{Édition électronique}

URL : https://journals.openedition.org/archeosciences/4298

DOI : $10.4000 /$ archeosciences. 4298

ISBN : 978-2-7535-3691-3

ISSN : 2104-3728

Éditeur

Presses universitaires de Rennes

\section{Édition imprimée}

Date de publication : 30 novembre 2014

Pagination : 235-246

ISBN : 978-2-7535-3689-0

ISSN : $1960-1360$

Référence électronique

Jimmy Linton, « Modéliser la gestion des grandes lames en silex de la fin du Néolithique à partir de la quantification des traces d'utilisation", ArcheoSciences [En ligne], 38 | 2014, mis en ligne le 30

novembre 2016, consulté le 01 février 2022. URL : http://journals.openedition.org/archeosciences/ 4298 ; DOI : https://doi.org/10.4000/archeosciences.4298 


\title{
Modéliser la gestion des grandes lames en silex de la fin du Néolithique à partir de la quantification des traces d'utilisation
}

\author{
Modelling the Management of Late Neolithic Large Flint Blades \\ through Use-Wear Quantification
}

\author{
Jimmy LinTON $^{\mathrm{a}}$
}

\begin{abstract}
Résumé : Ce travail est consacré à la caractérisation des modalités de gestion et d'utilisation des grandes lames en silex de la fin du Néolithique. Il s'agit de montrer qu'il est possible d'élaborer des modèles de gestion des lames à travers les données tracéologiques et technologiques. L'étude est fondée sur une approche intégrée des industries lithiques qui prend en compte l'identification des modes de production des supports, la typologie des pièces et les données fonctionnelles. Le modèle de gestion est mis en évidence grâce à une méthode précise d'analyse qui combine les données qualitatives de l'observation des stigmates d'utilisation et des supports avec les données métriques des outils d'ordre quantitatif. L'application de cette méthode a permis de discuter le modèle de fonctionnement des grandes lames qui avait été précédemment proposé. Les résultats obtenus démontrent que les grandes lames de la fin du Néolithique étaient des outils multifonctionnels qui faisaient l'objet d'une gestion sur le long terme.

Abstract: This work is dedicated to the characterization of late Neolithic large fint blades use and management. The question is to know if it is possible to develop a model of flint blades management using functional and technological data. The study is based on an integrated approach of lithic industries that takes into account the identification of productions methods, the tool typology and the use-wear analyses data. The developed model is highlighted by a precise method of analysis that combines the qualitative data from use-wear observation and the quantitative data from tool size. The application of this method allowes to explore and discuss the model that had been previously proposed. The results show that late Neolithic flint blades were multi-functional tools and were subject of a management over the long term.
\end{abstract}

Mots clés : tracéologie, technologie lithique, lames en silex, Néolithique final, Grand-Pressigny

Keywords: use-wear analysis, lithic technology, flint blades, late Neolithic, Grand-Pressigny

\section{INTRODUCTION}

Parmi les vestiges archéologiques de la préhistoire récente, l'outillage en silex, sa diversité et son évolution sont des sujets de recherche et de débats privilégiés. Depuis la fin du $\mathrm{xx}^{\mathrm{e}}$ siècle, l'essor des études technologiques, pétrographiques et tracéologiques permet d'observer le fonctionnement des industries lithiques préhistoriques. La tracéologie est une méthode d'étude de la fonction technique des outils à partir de l'analyse des usures au microscope. Elle permet notamment de participer à la description des différentes stratégies de gestion de l'outillage.

La fin du Néolithique constitue une période de mutations socio-économiques importantes. Des changements sont observés au sein du système technique, et particulièrement dans les industries lithiques. En effet, à partir de la fin du

${ }^{a}$ UMR6298 ARTeHIS, Membre associé, Université de Bourgogne - CNRS, 6 bd Gabriel 21000 DIJON. 
quatrième millénaire av. J.-C. se développent des ateliers de débitage de très grandes lames. Une partie de cette production est alors destinée à circuler sur de grandes distances, au-delà des limites géographiques des groupes culturels producteurs. Fréquemment considérées comme l'aboutissement de millénaires d'évolution des techniques de taille du silex, les productions de grandes lames sont aussi considérées comme la manifestation de profonds changements économiques et sociaux. En illustrant la circulation massive de biens sur de grandes distances et un artisanat spécialisé, les grandes lames sont souvent interprétées comme les marqueurs identitaires d'une élite émergente. Néanmoins, plusieurs études tracéologiques ont montré que les grandes lames étaient presque systématiquement marquées par des stigmates d'utilisation et qu'il s'agissait donc d'outils utilisés pour diverses tâches ordinaires. En même temps, les productions domestiques d'outils se simplifient au profit d'un essor important de la retouche des supports, pour le façonnage, mais aussi pour l'affûtage des tranchants. Il est alors très fréquent d'observer des pièces marquées par de nombreuses traces d'utilisation, révélant ainsi une partie de l'histoire technique complexe de chaque outil. D'un point de vue tracéologique, cela se traduit par des altérations de traces, par des stigmates incomplets, ou par une surimposition d'usures.

\section{INTÉRÊT DES PRODUCTIONS PRESSIGNIENNES ET CHOIX DES CORPUS ÉTUDIÉS}

Parmi les productions de grandes lames de la fin du Néolithique, les productions de la région du GrandPressigny (Indre-et-Loire) sont un des exemples les plus emblématiques et les mieux connus. Certains outils en silex du Grand-Pressigny, comme les poignards ou les racloirs à encoches, sont d'ailleurs des éléments qui contribuent à caractériser les ensembles du Néolithique final d'une partie de l'Europe occidentale. Plusieurs raisons font des productions pressigniennes le laboratoire d'étude le plus complet pour comprendre le phénomène de production de grandes lames de la fin du Néolithique. Les nombreuses pièces exportées au Néolithique ont fait l'objet d'un inventaire détaillé (Mallet et al., 2009). Ce corpus se répartit sur un très large territoire, et parmi des groupes culturels variés. En effet, l'importance des distances sur lesquelles les outils en silex du Grand-Pressigny ont circulé est une des caractéristiques les plus spectaculaires du phénomène pressignien. L'aire de diffusion couvre un territoire qui s'étend sur environ $1300 \mathrm{~km}$ du nord au sud et environ $1000 \mathrm{~km}$ d'est en ouest. La découverte la plus lointaine provient des rives de l'estuaire de la Weser, en Allemagne, au sein d'assemblages de la culture Cordé, à environ $900 \mathrm{~km}$ des ateliers de débitage. Vers l'Ouest, les exportations sont connues jusqu'au littoral atlantique, vers le sud elles sont signalées jusque dans le piémont pyrénéen et le delta du Rhône. Vers l'Est, l'arc alpin, le Rhin et la Weser semblent matérialiser la limite de diffusion.

En s'appuyant sur la résolution chronologique très fine des stratigraphies lacustres nord-alpines, une périodisation de la production de grandes lames a été proposée. Il existe de fortes présomptions d'une production de lames dès le Néolithique moyen. Ce n'est cependant qu'à partir de la fin du quatrième millénaire que la production de grandes lames en partie destinées à être aménagées en poignards semble réellement se mettre en place (Ihuel, 2009). Quelques pièces importées sont en effet connues dans des ensembles de la phase ancienne du groupe de Clairvaux de la station littorale de Chalain 4 (Fontenu, Jura). Ces ensembles sont datés de 3010 à 2990 av. J.-C. par dendrochronologie (Viellet, 2009). C'est toutefois à partir du $29^{\mathrm{e}}$ siècle que la phase de production massive commence. Cette étape, datée entre 2800 et 2400 avant notre ère et parfois appelée " phase classique ", correspond à l'apogée des ateliers de taille de la région du Grand-Pressigny, aussi bien d'un point de vue qualitatif que quantitatif. En effet, cette phase correspond à l'utilisation de la méthode livre-de-beurre pour le débitage de très grandes lames. Là aussi, les stratigraphies des stations lacustres nord-alpines servent de référence pour dater précisément ces productions.

Les pièces encore emmanchées retrouvées dans des stations lacustres comme Charavines en Isère (Bocquet, 1974) sont des arguments indiscutables de l'emploi de ces grandes lames emmanchées en poignards. D’une manière générale, la forme losangique de certains poignards et les premiers résultats tracéologiques semblent montrer qu'ils ont été utilisés dans un sens, puis dans un autre, pour optimiser le tranchant disponible. Il est alors impossible de distinguer la partie emmanchée de la partie active. De plus, la présence de nombreux fragments de grandes lames aménagés en outils $\mathrm{du}$ fond commun semble montrer que les recyclages après fragmentation étaient courants. Les fréquents affutages des tranchants par retouche semblent jouer aussi un rôle important dans la grande variabilité typo-morphologique des poignards, comme l'a proposé D. Ramseyer (Ramseyer, 1987) dans son étude du site de Delley-Portalban II (Fribourg, Suisse). Le polymorphisme des poignards et de leurs dérivés (figure 1) serait donc autant le résultat de traditions typoculturelles, que d'un mode particulier de gestion des tranchants des grandes lames, qui implique recyclage et affutage par la retouche. Mais comment s'organisait cette gestion des tranchants? Suivait-elle une règle? 

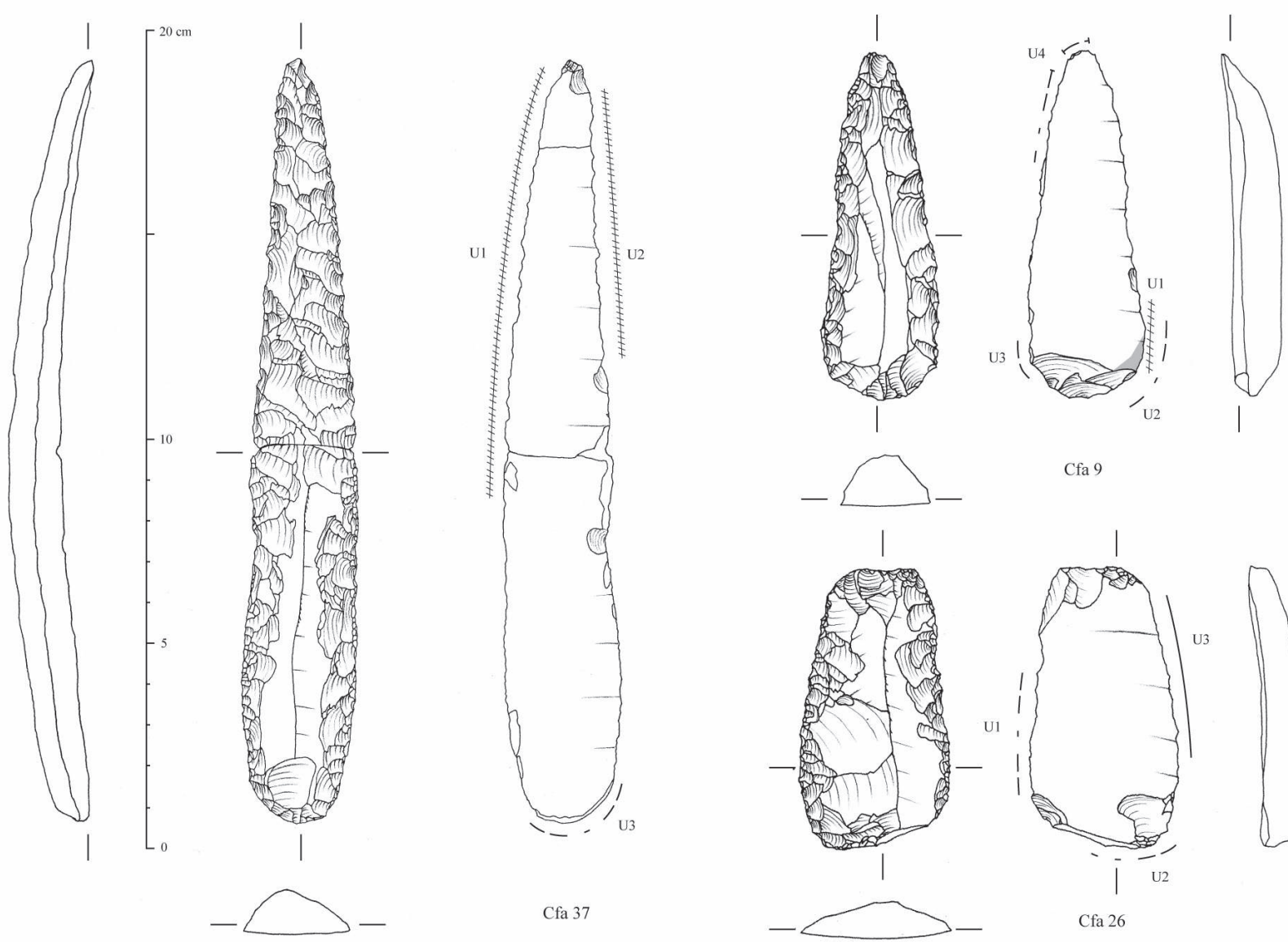

Cfa 9
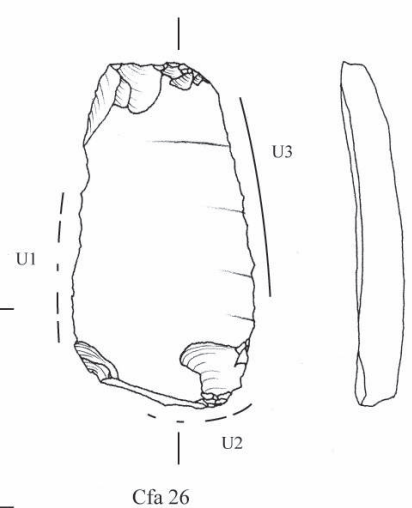

Figure 1 : Exemple d'outils sur grandes lames en silex du Grand-Pressigny (Chassey-le-Camp, collection Loydreau). Cfa 37 : poignard fortement réduit par la retouche (u1, action longitudinale sur des végétaux siliceux; u2, action longitudinale sur une matière tendre indéterminée; u3, action transversale sur une matière minérale type briquet). Cfa 9 : fragment de lame recyclée en outil (u1, action longitudinale sur des végétaux siliceux type céréales; u2, u3 et u4, action transversale sur une matière abrasive). Cfa 26 : fragment de lame recyclée en outil (u1, action transversale sur matière indéterminée; u2, action transversale sur matière abrasive; u3, action longitudinale sur matière abrasive). Ombrages M. Brunet.

Figure 1: Example of tools shaped from long blades made with upper Turonian flint from Grand-Pressigny area (Chassey-le-Camp, Loydreau collection). Cfa37: dagger significantly reduced by resharpening retouches (u1, siliceous plants cut in longitudinal motion; u2, unknown soft matter cut in longitudinal motion; u3, strike-a-light microwear in transversal motion). Cfa 9: tool made from a recycled blade fragment (u1, siliceous plants/cereals cut in longitudinal motion; $u 2, u 3$ and $u 4$, abrasive microwear in transversal motion). Cfa 26: tool made from a recycled blade fragment (u1, unknown matter microwear in transversal motion; u2, abrasive microwear in transversal motion; u3, abrasive microwear in longitudinal motion). Ink shading by Mickaël Brunet.

À la suite de travaux qui démontraient l'existence de plusieurs étapes dans l'utilisation des poignards pressigniens (Ramseyer, 1987; Mallet, 1992; Honegger, 2001), une étude tracéologique de divers ensembles de l'aire de diffusion (Beugnier et Plisson, 2004) a été réalisée. Cette étude a permis de proposer un modèle de gestion des poignards pressigniens en quatre stades et va servir de référence. Dans ce modèle, chaque stade correspond « à un état de réduction particulier des instruments et à un cortège d'usures spécifiques" (ibid., p. 147).
Pour mieux comprendre la gestion sur le long terme des grandes lames de la fin du Néolithique, un échantillon d'outils en silex du Grand-Pressigny a été sélectionné. L'échantillonnage a été réalisé dans le cadre d'un transect est/ ouest entre l'aire de production en Touraine et la région des Trois Lacs en Suisse (figure 2). Cet échantillonnage permet à la fois d'intégrer différents groupes culturels importateurs, mais aussi de travailler à l'échelle du réseau de circulation, de la zone de production jusqu'aux sites consommateurs très éloignés. Il a aussi le mérite d'intégrer des séries lacustres 


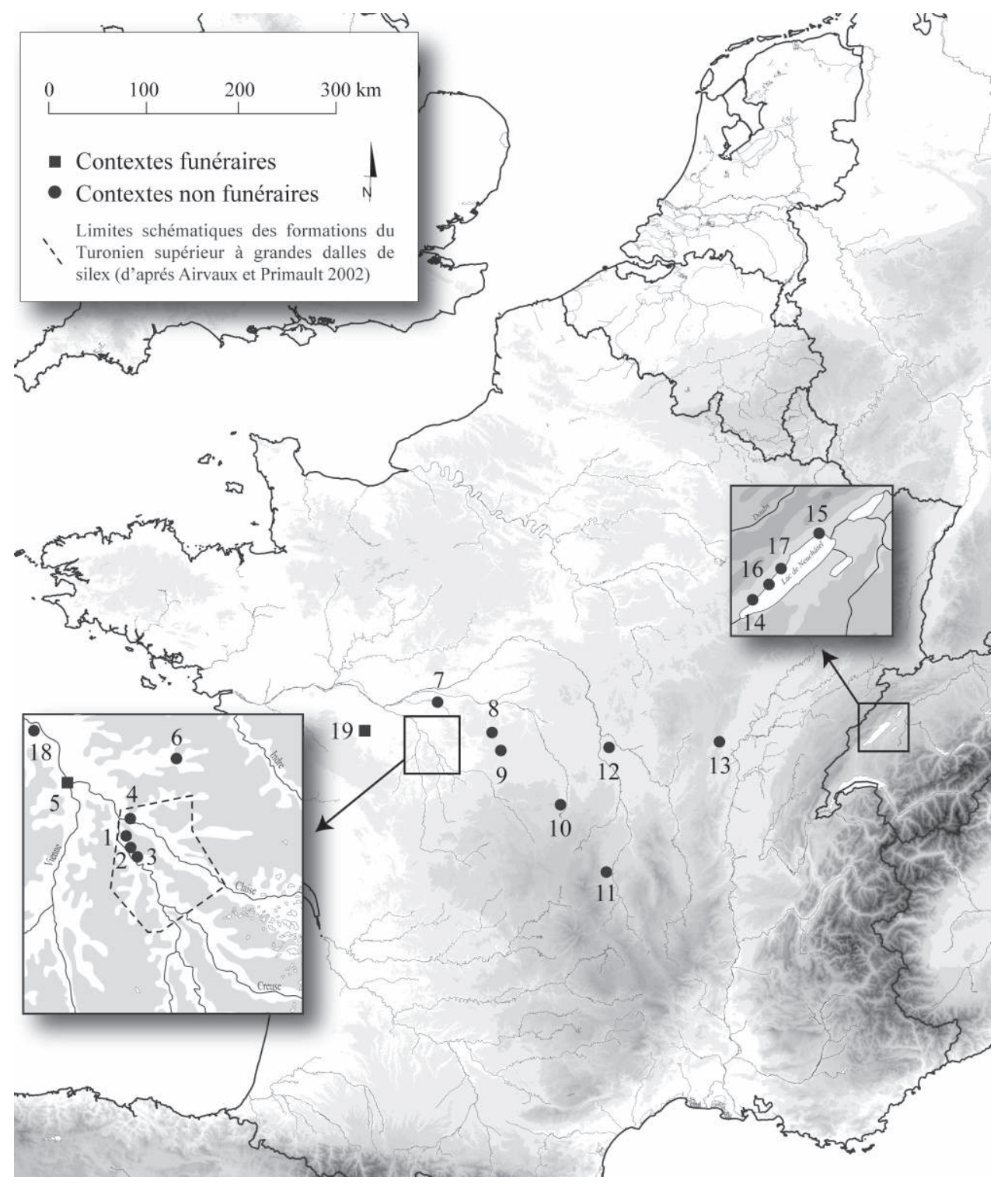

Figure 2 : Carte des ensembles échantillonnés pour l'étude. 1 : Bergeresse (Abilly, Indre-et-Loire); 2 : La Creusette (Barrou, Indre-et-Loire); 3 : Les Marais (Barrou, Indre-et-Loire) ; 4 : Le Foulon (Abilly, Indre-et-Loire) ; 5 : Le Bec des deux Eaux (Port sur Vienne, Indre-et-Loire); 6 : Les Sables de Mareuil (Ligueuil, Indre-et-Loire) ; 7 : Les Vignes de Saint Blaise (Truyes, Indre-et-Loire) ; 8 : Les Vaux (Moulins-surCéphons, Indre) ; 9 : Le Montet (Déols, Indre); 10 : Le Bournadiau (Quinssaines, Allier); 11 : La Croix de Saint-Roch (Le Crest, Puy-deDôme); 12 : La Chaume Vieille (Saint-Parize-Le-Chatel, Nièvre); 13 : Le Camp de Chassey (Chassey-le-Camp, Saône-et-Loire); 14 : Sous Colachoz (Concise, Suisse, VD); 15 : Bain des Dames (Saint Blaise, Suisse, NE); 16 : La Pièce du Prézault-La Varenne (Parçay-sur-Vienne, Indre-et-Loire) ; 17 : Découverte isolée à Crouzilles (Indre-et-Loire); 18 : Découverte isolée à Panzoult (Indre-et-Loire); 19 : Puyraveau Dolmen II (Saint-Léger-de-Montbrun, Deux-Sèvres).

Figure 2: Map of the sampled assemblages. 1 : Bergeresse (Abilly, Indre-et-Loire, France); 2: La Creusette (Barrou, Indre-et-Loire, France); 3: Les Marais (Barrou, Indre-et-Loire, France); 4: Le Foulon (Abilly, Indre-et-Loire, France); 5: Le Bec des deux Eaux (Port sur Vienne, Indreet-Loire, France); 6: Les Sables de Mareuil (Ligueuil, Indre-et-Loire, France); 7: Les Vignes de Saint Blaise (Truyes, Indre-et-Loire, France); 8: Les Vaux (Moulins-sur-Céphons, Indre, France); 9: Le Montet (Déols, Indre, France); 10: Le Bournadiau (Quinssaines, Allier); La Croix de Saint-Roch (Le Crest, Puy-de-Dôme, France); 12 La Chaume Vieille (Saint-Parize-Le-Chatel, Nièvre, France); 13: Le Camp de Chassey (Chassey-le-Camp, Saône-et-Loire, France); 14: Sous Colachoz (Concise, Vaud canton, Switzerland); 15: Bain des Dames (Saint Blaise, Neuchâtel canton, Switzerland); 16: Chemin du Puits Neuf (Langlade, Gard); 17: Isolated finding in Panzoult (Indre-et-Loire); 18: Isolated finding in Crouzilles (Indre-et-Loire); 19: Port-Conty ancient finding (Saint-Aubin-Sauges, Neuchâtel canton, Switzerland); 20: Bevaix, ancient finding (Neuchâtel canton, Switzerland); 21: Puyraveau Dolmen II (Saint-Léger-de-Montbrun, Deux-Sèvres, France); 22: La Pièce du Prézault-La Varenne (Parçay-sur-Vienne, Indre-et-Loire, France). 
nord-alpines afin de profiter de leur contexte exceptionnel. Léchantillon sélectionné se répartit entre 19 sites (figure 2). Il comprend 415 grandes lames, poignards, et dérivés de poignards en silex du Turonien supérieur de la région du Grand-Pressigny. Toutes les pièces ont fait l'objet d'un examen tracéologique approfondi qui a permis d'observer 696 usures. Tous les ensembles se répartissent chronologiquement entre le dernier quart du quatrième millénaire et le $25^{\mathrm{e}}$ siècle avant notre ère.

\section{MÉTHOde : OBSERVER, CARACTÉRISER, ET QUANTIFIER LES OUTILS EN SILEX ET LEURS STIGMATES D'UTILISATION}

La méthode mise en œuvre vise à caractériser la gestion des outils réalisés sur grandes lames. Pour cela, une approche intégrée de l'outillage a été mise en place en combinant les données tracéologiques aux données technologiques, typométriques, et typologiques.

L'analyse technologique permet d'appréhender un objet comme le résultat de procédés techniques de fabrication et/ ou d'utilisation. La succession des gestes techniques mis en œuvre forme une séquence technologique, la chaîne opératoire. Elle permet de replacer l'objet étudié au sein d'une suite de processus "allant de l'approvisionnement en matière première jusqu'à son abandon, en passant par toutes les étapes de fabrication et d'utilisation d'un outillage " (Inizan et al., 1995, 14). L'analyse typologique permet d'identifier les différentes classes d'outils et sert donc à décrire la composition de l'outillage au sein d'un assemblage.

La tracéologie est une méthode d'analyse qui vise à mettre en évidence la fonction technique et le fonctionnement des outils en étudiant les traces d'utilisation à l'aide de matériel de microscopie. Elle fait appel à un corpus de traces sur des outils expérimentaux qui servent de références pour interpréter les stigmates observés sur les outils archéologiques. Couplées aux résultats des deux premiers types d'examens, les données tracéologiques recueillies servent alors de base à une analyse intégrée de l'outillage en silex.

\section{Les tris technologiques et typologiques}

Les différents types de produits de débitage ont été classiquement distingués en cinq catégories qui ne seront pas précisément définies en raison de leur caractère conventionnel (ibid.) : les nucléus, les éclats, les lame(lle)s, les plaquettes et les déchets (débris, esquilles, cassons).

De même, les critères classiques de détermination ont été utilisés afin de distinguer les grands types de support (produits laminaires, éclats et plaquettes). Le tri a donc été effectué en fonction de différents paramètres, comme l'extension du cortex, le sens des enlèvements ou la fragmentation. De plus, l'allure des supports, les types de talon et les éventuelles préparations ont également été pris en compte afin d'affiner la détermination technologique. Dans certains cas, des éléments plus précis sur les modalités de production ont pu être pris en compte, comme le type de percussion ou le style de débitage. Les éléments issus de productions spécifiques comme la méthode livre-de-beurre ont fait l'objet d'une description particulière. Sur la base de ces critères de détermination, huit grands types de supports en silex du Grand-Pressigny ont été distingués :

- lames de livre-de-beurre

- grandes lames à talon non piqueté

- petites lames

- lames indéterminées

- lamelles

- éclat de mise en forme de livre-de-beurre

- éclat d'épannelage de livre-de-beurre

- éclats indéterminés.

D'un point de vue typologique, la démarche classificatoire utilisée s'inspire du répertoire de D. Binder (1987), repris plus récemment sous différentes variantes, adaptées à des contextes régionaux (Honegger, 2001; Perrin, 2003; Léa, 2004). Ce type de classification a l'avantage de prendre en compte la nature du support et la technique de façonnage en plus de la morphologie des zones retouchées de l'outil. En tenant compte de ces critères, le répertoire typologique se décline alors en groupes, classes, types et sous-types. À partir de l'observation des éléments lithiques qui composent le corpus, le répertoire typologique a été subdivisé en 11 groupes. Concernant les produits laminaires, on notera que le groupe des pièces brutes ou à enlèvements marginaux correspond aux éléments dénués de retouche, ou affectés par une retouche marginale irrégulière. Le groupe des lames à retouche (bi)latérale réunit les éléments laminaires aménagés par une retouche qui affecte un ou les deux bords. Les extrémités peuvent être brutes ou aménagées; les éléments dont une des extrémités est appointée sont considérés comme des poignards, à l'exception des plus petits éléments, considérés a priori comme perçoirs.

\section{La caractérisation des stigmates}

\section{Méthodes d'observation, de description et interprétation des stigmates d'utilisation}

La méthode employée combine classiquement l'observation à faible grossissement pour l'étude des stigmates à échelle macroscopique, et leurs associations, et l'observa- 
tion à fort grossissement pour l'étude plus fine des polis (Semenov, 1964; Tringham et al., 1974; Keeley, 1980; Van Gijn, 1989; González Urquijo et Ibáñez Estévez, 1994, Gassin, 1996). La phase analytique s'inspire très largement de celle mise en place sur des séries du Chasséen provençal par B. Gassin (Gassin, 1996). Elle consiste à intégrer les résultats tracéologiques à une étude technologique et typologique, à établir des spectres fonctionnels quantifiés et à interpréter les résultats dans une perspective socio-économique.

Les supports laminaires de grande taille et les pièces façonnées de la fin du Néolithique font fréquemment l'objet d'utilisations multiples, dans le cadre d'une gestion à long terme de l'outillage. C'est pourquoi le volet tracéologique de la base de données permet de renseigner chaque zone utilisée indépendamment à travers sept champs, dont cinq sont de nature qualitative (les deux derniers champs sont déjà des données interprétatives) :

- la position de la zone utilisée sur la pièce (bord droit par exemple);

- le type de zone active utilisée (front de grattoir par exemple);

- l'intégrité de la zone utilisée renseigne si elle est recoupée par une retouche ou une utilisation postérieure;

- la cinématique d'utilisation correspond au geste effectué et au fonctionnement de l'outil lors de son utilisation;

- le champ « matière » renseigne sur le type de matériaux travaillés.

Deux autres champs de nature quantitative ont également été pris en compte, la longueur de la zone utilisée et son extension transversale.

\section{De l'observation à l'interprétation, les limites de la tracéologie}

Afin d'interpréter les stigmates observés sur les pièces archéologiques, la méthode d'analyse tracéologique nécessite la constitution de référentiels expérimentaux et le recours à des comparaisons ethnographiques. Ainsi, il est possible de proposer des gestes effectués et des matières travaillées par les préhistoriques à l'origine des ensembles archéologiques étudiés.

En effet, combinés entre eux, les différents stigmates d'utilisation observés à la surface des outils en silex forment un faisceau d'indices qu'il faut interpréter. Certaines traces sont assez caractéristiques et permettent de remonter rapidement au fonctionnement de l'outil, et, dans le meilleur des cas jusqu'à la matière travaillée. C'est le cas par exemple de la coupe de céréales. Cependant, les diagnostics atteignent des niveaux d'interprétation différents selon les degrés possibles de lecture, et la précision des informations. Plusieurs facteurs peuvent être des obstacles à une détermination précise : faible intensité d'utilisation, altération des stigmates, texture grenue du silex, programme expérimental incomplet... La caractérisation se cantonne alors à un sens d'utilisation probable et un degré de dureté de la matière travaillée. Le fonctionnement de l'outil et la matière sur laquelle il a travaillé permettent ensuite, dans le meilleur des cas, d'établir un geste technique et de faire le lien avec une activité artisanale ou domestique. Il faut alors tenter de replacer cette action dans une chaîne opératoire, en croisant ces données avec les résultats d'autres disciplines comme l'archéozoologie ou la carpologie. Ces restitutions sont le plus souvent incomplètes ou hypothétiques, mais elles permettent tout de même d'établir des modèles que l'expérimentation peut alors vérifier.

\section{Les différentes usures rencontrées}

La description des principales usures rencontrées sur les outils archéologiques lors de l'analyse a été synthétisée dans le tableau 1. Les principaux critères de caractérisation et de différenciation utilisés dans le cadre de ce travail sont exposés. Ces critères, communément admis par les autres tracéologues, sont issus de nombreuses expérimentations exploratoires et des travaux des autres chercheurs en tracéologie.

Lorsque les stigmates ne sont pas assez caractéristiques, trop peu marqués ou masqués par des altérations, la reconnaissance des matériaux travaillés n'est pas possible. Il est tout de même envisageable dans de nombreux cas, d'estimer la dureté du matériau travaillé et la cinématique d'utilisation. En toute logique, il est préférable de rester prudent dans les attributions en l'absence de critère réellement diagnostique. Les traces observées sont le plus souvent des écaillures et enlèvements d'utilisation. La quantité, la morphologie, les dimensions et l'organisation des écaillures sont des indices pour déterminer la dureté des matériaux et la cinématique d'utilisation. Dans certains cas, un poli marginal difficile à distinguer du " bruit " des altérations est visible. Dans d'autres cas un émoussé du tranchant, et parfois des nervures de la face supérieure, sont pris en compte.

La surimposition des traces d'utilisation produit une sorte d'effet palimpseste qui entraîne deux types de problèmes. En premier lieu, il faut souligner que les stigmates sont plus difficiles à identifier sur les pièces qui font l'objet d'usages multiples et d'une gestion à long terme, que sur celles à usage unique sans gestion par la retouche. Il devient alors difficile, dans certains cas, de différencier les usures superposées. En conséquence, la quantification des stigmates peut devenir imprécise et nécessite le développement d'une méthode adaptée à ce contexte tracéologique particulier. Cette situation pose d'autant plus de problèmes, que les résultats fonctionnels seront comparés avec ceux du reste 


\begin{tabular}{|c|c|c|}
\hline & Longitudinale & Transversale \\
\hline $\begin{array}{l}\text { Abrasive / } \\
\text { souple } \\
\text { abrasive }\end{array}$ & $\begin{array}{l}\text { Poli brillant à peu brillant bifacial, modelé doux, à } \\
\text { trame serrée et limites floues irrégulières } \\
\text { Stries peu profondes mal délimitées } \\
\text { Emoussé du tranchant } \\
\text { Ecaillement irrégulier adouci par le poli }\end{array}$ & $\begin{array}{l}\text { Poli brillant à peu brillant à trame serrée et limites floues } \\
\text { irrégulières } \\
\text { Stries peu profondes mal délimitées } \\
\text { Fort émoussé asymétrique du tranchant }\end{array}$ \\
\hline $\begin{array}{c}\text { Tissu animal } \\
\text { résistant }\end{array}$ & $\begin{array}{l}\text { Poli fluide peu brillant aux limites diffuses, discret } \\
\text { Ecaillements bifaciaux ou alternants discontinus } \\
\text { Fil du tranchant adouci }\end{array}$ & Non rencontrée \\
\hline $\begin{array}{l}\text { Végétaux } \\
\text { siliceux type } \\
\text { céréales }\end{array}$ & $\begin{array}{l}\text { Poli brillant envahissant bifacial à trame compacte, } \\
\text { modelé dur et limites nettes irrégulières, parfois } \\
\text { ondulé, nombreux trous } \\
\text { Ecaillements bifaciaux ou alternants } \\
\text { Fines stries comblées continues ou en pointillé }\end{array}$ & Non rencontrée \\
\hline $\begin{array}{l}\text { Végétaux } \\
\text { siliceux }\end{array}$ & $\begin{array}{l}\text { Poli brillant envahissant bifacial à trame compacte, } \\
\text { modelé dur et limites nettes } \\
\text { Ecaillements bifaciaux ou alternants }\end{array}$ & $\begin{array}{l}\qquad \text { "Type } 23 \text { " } \\
\text { Poli brillant marginal asymétrique à trame compacte et } \\
\text { limites nettes irrégulières } \\
\text { Emoussé en biseau arrondi peu marqué }\end{array}$ \\
\hline $\begin{array}{l}\text { Végétal } \\
\text { ligneux }\end{array}$ & $\begin{array}{l}\text { Poli plat brillant de trame ouverte à fermée, } \\
\text { Ecaillements bifaciaux importants }\end{array}$ & $\begin{array}{l}\text { Poli brillant à trame très ouverte, coalescence qui s'étire en } \\
\text { fonction du relief } \\
\text { Ecaillement important sur tranchant à angle aigu } \\
\text { Emoussé asymétrique }\end{array}$ \\
\hline $\begin{array}{l}\text { Végétaux } \\
\text { indéterminés }\end{array}$ & $\begin{array}{l}\text { Poli brillant à trame ouverte } \\
\text { Ecaillements bifaciaux ou alternants }\end{array}$ & $\begin{array}{l}\text { Poli brillant à trame ouverte } \\
\text { Emoussé asymétrique }\end{array}$ \\
\hline $\begin{array}{c}\text { Minérale } \\
\text { type briquet }\end{array}$ & Non rencontrée & $\begin{array}{l}\text { Tranchant ou extrémité fortement émoussé } \\
\text { Ecrasements et cupules adoucis par la forte usure abrasive } \\
\text { Peut se prolonger par un poli envahissant brillant de texture } \\
\text { plus ou moins rugueuse à trame compacte } \\
\text { Nombreuses stries d'orientation et de morphologie variés }\end{array}$ \\
\hline $\begin{array}{c}\text { Dure } \\
\text { animale }\end{array}$ & $\begin{array}{l}\text { Poli brillant lisse ou ondulé à trame compacte et aux } \\
\text { limites nettes à diffuses, à cannelures, stries ou des } \\
\text { plissures, parfois des « fissures », discontinu et } \\
\text { désorganisé } \\
\text { Emoussé discontinu } \\
\text { Ecaillements bifaciaux importants }\end{array}$ & $\begin{array}{l}\text { Poli brillant lisse ou ondulé à trame compacte et aux limites } \\
\text { nettes, à cannelures, stries ou des plissures, parfois } \\
\text { des «fissures » } \\
\text { Emoussé en biseau } \\
\text { Ecaillement asymétrique }\end{array}$ \\
\hline $\begin{array}{c}\text { Dure } \\
\text { indéterminée }\end{array}$ & $\begin{array}{l}\text { Ecaillements bifaciaux importants } \\
\text { Poli discontinu et désorganisé }\end{array}$ & $\begin{array}{l}\text { Ecaillements importants } \\
\text { Emoussé asymétrique }\end{array}$ \\
\hline $\begin{array}{c}\text { Tendre } \\
\text { indéterminée }\end{array}$ & $\begin{array}{l}\text { Ecaillements bifaciaux ou alternants } \\
\text { Fil du tranchant adouci }\end{array}$ & $\begin{array}{l}\text { Ecaillements adoucis } \\
\text { Emoussé asymétrique }\end{array}$ \\
\hline Indéterminée & Ecaillements alternants & $\begin{array}{l}\text { Ecaillements } \\
\text { Emoussé asymétrique }\end{array}$ \\
\hline
\end{tabular}

Tableau 1 : Description des principales usures rencontrées lors de l'étude. Table 1: Description of the main list of microwear found during the study. 
des assemblages afin d'établir des spectres fonctionnels quantifiés, qui permettront des interprétations dans une perspective socio-économique (Gassin, 1996). De plus, comme cela vient d'être souligné, les assemblages du Néolithique final sont systématiquement constitués d'outils importés à gestion sur le long terme, et d'outils issus de productions plus simples, destinés à une utilisation unique et souvent brève.

\section{RÉsultats : DE la QUANTIFICATION À LA RESTITUTION DU MODÈLE DE GESTION DES GRANDES LAMES}

\section{Mise à l'épreuve du premier modèle proposé}

Comme cela vient d'être souligné, les premières études technologiques et tracéologiques sur l'outillage laminaire pressignien ont mis en évidence plusieurs étapes dans l'utilisation et la gestion des tranchants des grandes lames. Ce type de gestion a d'abord été reconnu par les études typologiques (Ramseyer, 1987; Mallet, 1992), qui ont très tôt mis en évidence le polymorphisme typologique des poignards et grandes lames. La gestion sur le long terme des grandes lames pressigniennes a ensuite été abordée d'un point de vue tracéologique (Beugnier et Plisson, 2004), ce qui a amené les auteurs à proposer un premier modèle de gestion des poignards. Dans ce modèle, ils ont distingué quatre grandes étapes (tableau 2) dans la vie d'un poignard " correspondant, à la fois, à un état de réduction particulier des instruments et à un cortège d'usures spécifiques» (ibid..., p. 147) :

- Dans leur état initial, c'est-à-dire encore faiblement modifié par les retouches, les poignards sont utilisés pour la découpe de matière tendre carnée et de tiges végétales. Les stigmates sont dans tous les cas peu prononcés. Sur certaines pièces, des usures abrasives longitudinales sont associées à des gestes de manipulation, comme celui de fourrer le poignard dans un étui en cuir.

- Le second stade d'utilisation du modèle de Beugnier et Plisson correspond à des poignards qui ont déjà subi plusieurs cycles de retouche des tranchants «sans que leur silhouette et leur qualité tranchante initiales aient été altérées (poignards losangiques)» (Ibid., p. 147). Les stigmates associés à ce stade d'utilisation sont les lustres de matières végétales sous leur forme longitudinale et transversale. Selon les auteurs, il semble que les traces correspondent à une intensité d'utilisation plus importante qu'au stade précédent.

- Au troisième stade du modèle, "les poignards sont très sensiblement raccourcis, leur silhouette initiale a été largement altérée de diverses façons et certains bords actifs sont quasiment abrupts» (ibid., p. 149). Des lustres de matière végétale sont toujours observés, mais des stigmates d'utilisation comme briquet, ou relatifs au traitement des peaux font aussi leur apparition.

- Au dernier stade d'utilisation, les pièces sont fragmentées et ne correspondent plus typologiquement à des poignards : « les usures végétales initiales ne subsistent plus que sous des formes résiduelles que la retouche n'a pas emportées. Réduits à l'état de fragments, ils sont recyclés comme briquets ou outils à travailler les peaux " (ibid., p. 149).

Ainsi, dans ce modèle, à chaque stade typo-morphologique des poignards correspond une association d'usures spécifique.

J'ai testé ce modèle en le confrontant aux données acquises. Le premier obstacle a été de définir plus précisément les quatre stades typo-technologiques afin de faire correspondre les outils étudiés. Il faut souligner la difficulté pour établir une frontière typométrique nette entre les éléments des différents stades. Le quatrième stade pose le moins de problèmes, puisqu'il se compose de pièces fragmentées ou recyclées, quoique l'on peut se demander à quel stade asso-

\begin{tabular}{|c|c|c|}
\cline { 2 - 3 } \multicolumn{1}{c|}{} & État morpho-typologique & Usures associées \\
\hline Stade 1 & $\begin{array}{c}\text { Poignards au stade initial, peu } \\
\text { retouchés }\end{array}$ & $\begin{array}{c}\text { Découpe de matière tendre carnée et de tiges } \\
\text { végétales }\end{array}$ \\
\hline Stade 2 & $\begin{array}{c}\text { Poignards ayant fait l'objet de } \\
\text { plusieurs cycles de retouche }\end{array}$ & $\begin{array}{c}\text { Matières végétales en actions longitudinales } \\
\text { et transversales très prononcées }\end{array}$ \\
\hline Stade 3 & $\begin{array}{c}\text { Poignards raccourcis, } \\
\text { tranchants abrupts }\end{array}$ & $\begin{array}{c}\text { Usures végétales, usures abrasives et } \\
\text { utilisations en briquet }\end{array}$ \\
\hline Stade 4 & $\begin{array}{c}\text { Poignards fragmentés } \\
\text { recyclés en outils tiers }\end{array}$ & $\begin{array}{c}\text { Usures végétales résiduelles, usures } \\
\text { abrasives et utilisations en briquet }\end{array}$ \\
\hline
\end{tabular}

Tableau 2 : Modèle de corrélation entre la morpho-typologie des poignards et les usures associées, proposé par V. Beugnier et H. Plisson (2004).

Table 2: Correlative model between daggers morpho-typology and associated microwear, proposed by V. Beugnier et $H$. Plisson (2004). 
cier un fragment de poignard au premier stade, mais non réutilisé après une fracture. Il est difficile, voire impossible, de séparer les poignards entiers en trois groupes en fonction de leur degré de réduction, puisque la dimension (et donc leur degré de rétrécissement) est une variable continue. C'est pourquoi j'ai fait le choix de tester le modèle dans l'autre sens, en partant des données tracéologiques, en regroupant les outils par association de stigmates. L'homogénéité et la continuité typométrique des groupes ont alors été mises à l'épreuve simplement à partir de la dimension (longueurlargeur) des pièces qui les composent. Cette démarche, qui consiste à prendre en compte les données tracéologiques, permet de différencier les éléments supposés appartenir aux deux premiers stades du modèle. En effet, dans ce modèle, les pièces du premier stade sont affectées par des stigmates de découpe de matière tendre carnée et de tiges végétales. En revanche, celles du second stade sont marquées par des usures de matières végétales en actions longitudinales et transversales très prononcées. Les pièces appartenant aux stades trois et quatre sont marquées par le même type d'usures (usures végétales, usures abrasives et utilisations en briquet). C'est pourquoi elles n'ont pas pu être différenciées, à l'exception des pièces marquées par des usures plus variées. Ces dernières sont renvoyées au quatrième groupe d'association des stigmates, correspondant au stade 4 du modèle de V. Beugnier et H. Plisson.

La simple observation de la projection des pièces selon la longueur et la largeur (figure 3) amène à douter de l'évidence de chaque groupe. En effet la dimension des pièces qui les composent ne semble pas présenter de différences significatives, à l'exception du quatrième groupe qui semble se composer d'éléments de longueur plus restreinte.

Le tri par associations de stigmates n'est donc pas satisfaisant pour confirmer le modèle de V. Beugnier et $\mathrm{H}$. Plisson, les nuages de points étant superposables, à l'exception du nuage des pièces du groupe 4, toutes de faibles dimensions. Les données ont été mises à l'épreuve de tests statistiques (analyses de variance suivies de tests de Tukey). La proba-
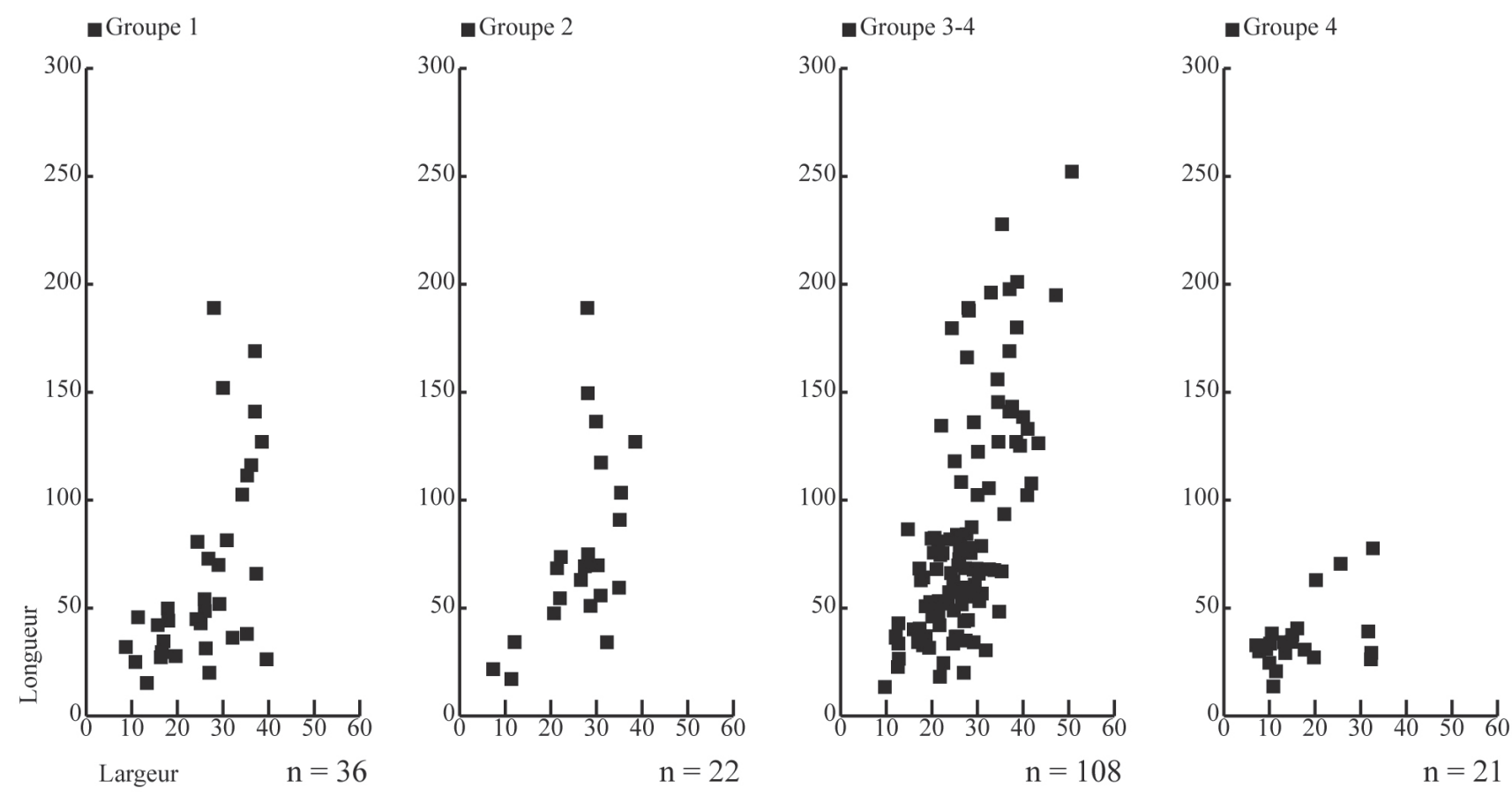

Figure 3 : Projection des poignards, dérivés de poignards et fragments de grandes lames à retouche bilatérale en silex du Grand-Pressigny selon leur longueur et leur largeur $(\mathrm{mm})$. Chaque graphique regroupe les pièces dont l'association de stigmates correspond aux stades d'utilisation des poignards selon le modèle proposé par V. Beugnier et H. Plisson (2004). On constate que les nuages ne se différencient guère à l'exception du groupe 4 qui s'individualise effectivement par la faible dimension des pièces qui le composent. Ce résultat permet de nuancer le modèle d'association d'usure et typo-morphologie proposé, notamment pour la différenciation entre les groupes 1 , 2 et 3 . Ce constat a été confirmé par des tests statistiques (analyses de variance suivies de tests de Tukey).

Figure 3: Scatter plot representing the length and width of long blades, daggers and fragments made with upper Turonian flint from GrandPressigny area. Each plot integrates flint tools grouped by microwear association. The associations correspond to V. Beugnier and H. Plisson (2004) daggers use stages model. Scatter plots 1, 2 and 3 don't show many differences. That means we have to qualify the V. Beugnier and H. Plisson microwear and typology association model. This result is confirmed by statistical tests (analysis of variance and Tukey's test). 
bilité critique obtenue par l'analyse de variance (ANOVA) étant inférieure à $5 \%$, au moins un des groupes diffère donc des autres de façon significative. La différence entre les groupes a alors été éprouvée à l'aide d'un test de Tukey. Il est apparu que seul le quatrième groupe diffère des autres de façon significative. L'existence des trois premiers groupes n'a donc pas été prouvée. Ainsi, ces analyses statistiques sont venues confirmer ce que l'on peut observer empiriquement sur les projections de nuage de point : seul le quatrième groupe d'associations de stigmates se démarque des trois autres d'un point de vue typométrique (longueur et largeur). Les pièces des groupes 1, 2 et 3 d'association de stigmates, ne se différencient pas, ce qui rejoint aussi les premières remarques sur la difficulté de différencier et classer les éléments en quatre stades distincts.

\section{Croisement des données métriques et tracéologiques}

J'ai alors cherché à savoir si l'utilisation des poignards, et de leurs dérivés, en silex pressigniens, était corrélée à la dimension des outils et fragments, indépendamment des groupes d'associations de stigmates proposés par $\mathrm{V}$. Beugnier et $\mathrm{H}$. Plisson. Pour cela, les dimensions des pièces triées par types d'usures ont été projetées. Seuls les cinq types de stigmates d'utilisation les plus fréquents ont été pris en compte, car ils représentent près de $80 \%$ de l'effectif des zones utili- sées observées. Dans un premier temps, toutes les pièces ont été prises en compte, même celles sur lesquelles les usures observées sont incomplètes. Les stigmates incomplets sont assez fréquents sur les grandes lames de la fin du Néolithique car elles font l'objet d'une gestion sur le long terme par le biais d'affûtage des tranchants et de recyclage après fragmentation. Les traces d'utilisations tronquées par une retouche postérieure ou une fracture sont donc fréquentes. De plus, ces pièces aux stigmates tronqués ne reflètent pas la dimension de l'outil au moment de son utilisation. En effet, certaines usures comme les traces d'action longitudinale sur une matière végétale siliceuse ont une persistance assez élevée. Elles peuvent ainsi encore être détectées même après plusieurs cycles de retouches et le recyclage de l'outil. En prenant en compte les outils dont les traces sont incomplètes, on peut remarquer qu'il n'y a pas de différences flagrantes (figure 4).

On observe tout de même une très légère tendance générale de différenciation des longueurs d'outils entre groupes d'usures. Le groupe des outils marqués par des usures abrasives longitudinales se distingue quelque peu par des longueurs plus importantes, et celui des outils marqués par des usures minérales de type briquet semble s'individualiser légèrement par des longueurs plus faibles.

Afin d'affiner les résultats, et de s'affranchir du biais des usures incomplètes, une seconde projection a été réalisée en comptabilisant uniquement les usures entières (figure 5). De

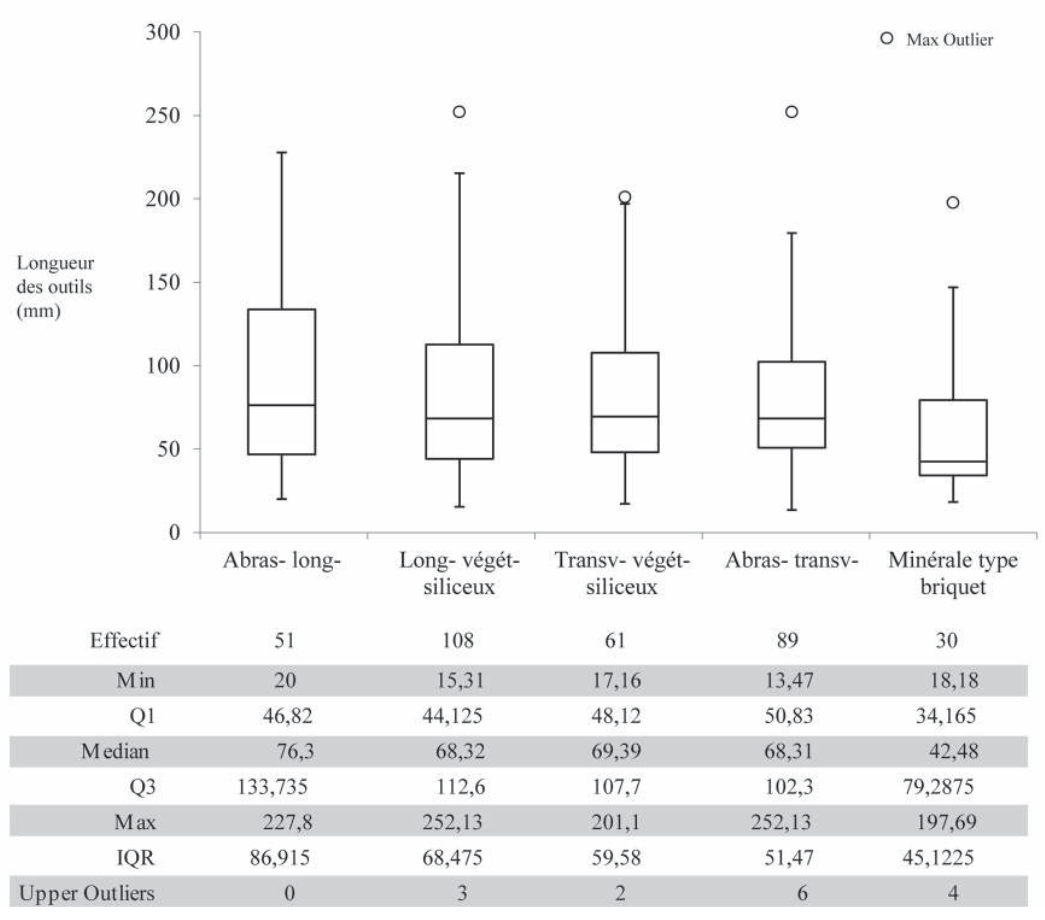

Figure 4 : Graphique " boxplot " représentant la longueur $(\mathrm{mm})$ des poignards, dérivés de poignards et grandes lames retouchées ou fragments (silex du Grand-Pressigny), regroupés par types d'usures. Seules les cinq usures les plus fréquentes ont été représentées. On constate que seul le groupe des outils marqués par des usures abrasives longitudinales se distingue légèrement par des longueurs plus importantes. En revanche, le groupe des outils marqués par des usures minérales de type briquet semble s'individualiser légèrement par des longueurs plus faibles. Certaines pièces sont présentes dans plusieurs catégories d'usures puisqu'elles sont marquées par différents types de stigmates.

Figure 4: Boxplot representing the length ( $\mathrm{mm}$ ) of long blades, daggers and fragments made with upper Turonian flint from Grand-Pressigny area, grouped by microwear. Only the five most common microwear are represented. The tools with abrasive microwear in longitudinal motion can be distinguished with higher length while tools with strike-alight mineral microwear can be distinguished with lower length. Tools with different microwear will thus be found in several groups of the boxplot. 
Figure 5 : Graphique «boxplot " représentant la longueur $(\mathrm{mm})$ des poignards, dérivés de poignards et grandes lames retouchées ou fragments (silex du Grand-Pressigny), regroupés par types d'usures. Seules les cinq usures les plus fréquentes sont représentées, et uniquement les usures entières. On constate une tendance nette de corrélation entre la longueur de l'outil et son utilisation. Les usures longitudinales sur des végétaux siliceux et les usures abrasives longitudinales semblent réservées aux pièces les plus longues, alors que l'utilisation comme briquet est essentiellement observée sur des pièces de faible dimension.

Figure 5: Boxplot representing the length $(\mathrm{mm})$ of long blades, daggers and fragments made with upper Turonian flint from Grand-Pressigny area, grouped by microwear. Only the five most common microwear are represented, and only the tools with complete one. There is a very clear correlation between tool length and use. Abrasive microwear and siliceous plants cut in longitudinal motion seem to be assigned to the longest tools. Conversely, strike-a-light microwear are mainly seen on shortest tools.

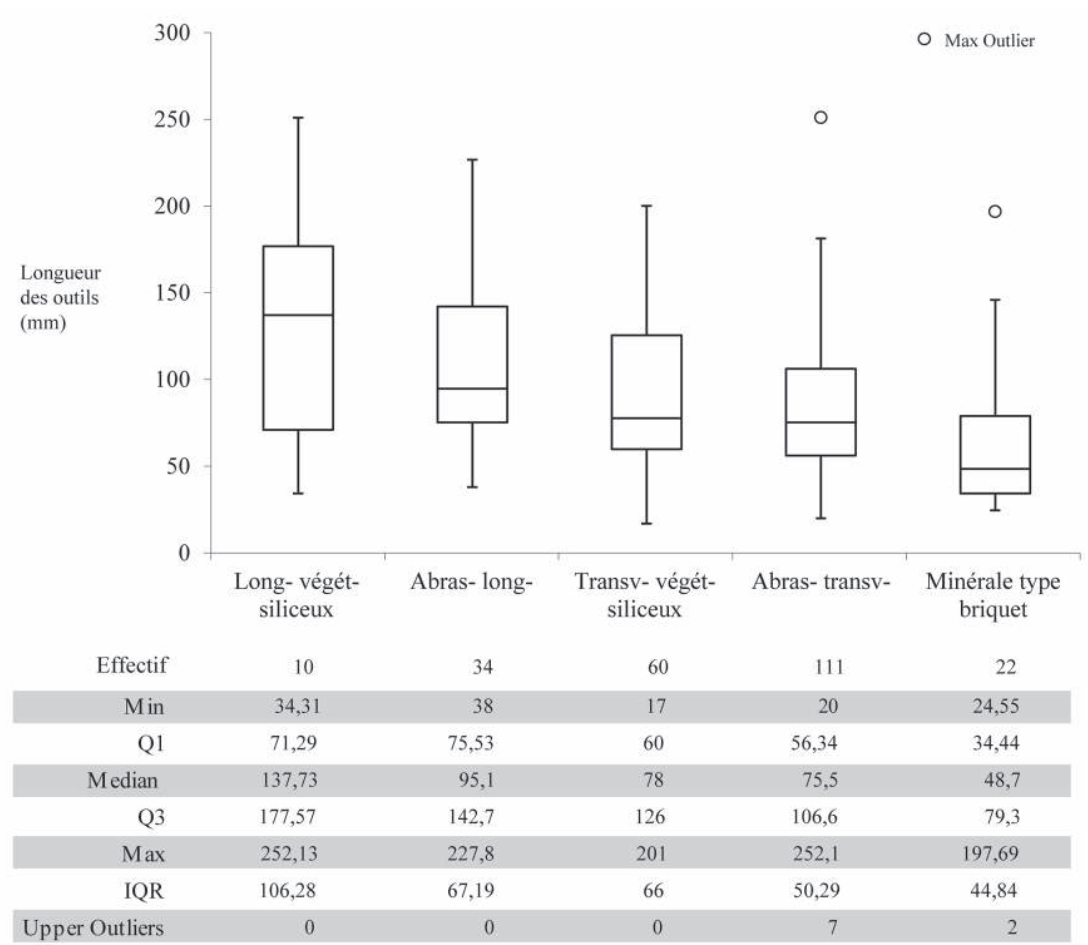

cette façon, la tendance est beaucoup plus nette, les groupes d'usures semblent se différencier par la longueur des outils mis en œuvre. Ainsi, le groupe des usures d'action longitudinale sur des végétaux regroupe le plus d'outils de longueur importante. Il est suivi par le groupe des outils marqué par des usures abrasives longitudinales. Enfin, le groupe d'usure qui rassemble les outils aux dimensions les plus faibles est celui des usures minérales de type briquet. Il faut tout de même souligner qu'il ne s'agit que d'une tendance statistique, on remarque que des outils de faible et grande dimension sont présents dans chaque groupe d'usure.

\section{Conclusion}

Les grandes lames en silex du Grand-Pressigny faisaient donc l'objet d'une gestion sur le long terme, une véritable économie du support, qui optimisait le tranchant disponible en adaptant l'usage à la longueur de lame disponible. Le tranchant disponible est en effet renouvelé et entretenu par le biais de retouches d'affûtage, et les recyclages d'outil sont très fréquents. Une tendance nette de corrélation entre type d'usures observées et longueur des pièces a pu être constatée. En effet, les utilisations pour des gestes de découpe en action longitudinale, comme la récolte de céréale, sont le plus fréquemment observées sur les lames encore longues et entières. Au contraire, les activités techniques ne nécessitant pas une lon- gueur de tranchant importante, comme le raclage des peaux ou l'utilisation en briquet, étaient le plus souvent réalisées avec de grandes lames raccourcies ayant déjà subi de nombreux cycles de retouche. Néanmoins, on a pu voir que ce modèle de gestion n'était pas exclusif. Des lames encore longues et peu retouchées pouvaient être employées en action transversale, et inversement, des lames déjà fortement retouchées pouvaient dans certains cas encore servir en action longitudinale. La réalité semble donc complexe, la destination fonctionnelle des poignards selon leur morphométrie et donc leur stade d'avivage/recyclage ne semble pas obéir à une règle rigide et linéaire, même si une tendance nette existe. La présente étude est donc un premier pas vers l'élaboration de modèles de gestion des outils en silex à partir de données quantifiées. Elle devra être étendue à d'autres contextes chronoculturels et d'autres productions de grandes lames.

\section{Remerciements}

J'exprime toute ma gratitude aux personnes qui m'ont fait confiance et donné accès à des collections : Ariane Winiger, Jérôme Bullinger, Matthieu Honegger, Pauline de Montmollin, Catherine Louboutin, Laure-Anne Millet Richard, Tony Hamon, Stéphane Bourne, Christian Verjux, Stella Weiser, Nicole Mallet, Damien Leroy, J.-B. Chimier, Sylvie Saintot, David Pelletier, Jean-Paul Thevenot et Brigitte Maurice-Chabard. 


\section{Bibliographie}

Beugnier V., Plisson H., 2004. Les poignards en silex du Grand-Pressigny : fonction de signe et fonctions d'usage. In P. Bodu, C. Constantin (dir.). Approches fonctionnelles en préhistoire, Actes du XXV Congrès Préhistorique de France (24-26 novembre 2000, Nanterre). Publié par la Société Préhistorique Française : 139-154.

Binder D., 1987. Le Néolithique ancien provençal : typologie et technologie des outillages lithiques. Supplément à Gallia Préhistoire, 24. Paris, éditions du CNRS.

Bocquet A., 1974. Les poignards néolithiques de Charavines (Isère) dans le cadre de la civilisation Saône-Rhône. Études Préhistoriques, $9:$ 7-17.

Gassin B., 1996. Évolution socio-économique dans le Chasséen de la grotte de l'Église supérieur (Var). Apport de l'analyse fonctionnelle des industries lithiques. Monographie du CRA, 17, Paris, éditions du CNRS.

Gonzales Urqijo J.M., Ibanez Estevez J.J., 1994. Metodologia de analisis funcional de instrumentos tallados en silex. Cuadernos de Arqueologia, 14, Universidad de Deusto, Bilbao.

Honegger M., 2001. L'industrie lithique taillée du Néolithique moyen et final de Suisse. Monographie du CRA, 17, Paris, éditions du CNRS.

Inuel E., 2009. De la circulation des lames à la circulation des poignards : mutation des productions lithiques spécialisées dans l'Ouest de la France du ve au III millénaire. Thèse de doctorat, Université de Paris X-Nanterre.

Inizan M.-L., Reduron M., Roche H. Tixier J., 1995. Technologie de la pierre taillée. Préhistoire de la pierre taillée, 4, Meudon, CREP.

Keeley L.-H., 1980. Experimental determination of Stone Tool Uses, a microwear analysis. University of Chicago press, Prehistoric Archeology and Ecology Series.
Mallet N., Ihuel E., Genty P., Richard G., DelcourtVlaeminck M., Verjux C., Weisser S., 2009. La diffusion des silex du Grand-Pressigny au Néolithique récent et final : nouvel état de l'inventaire et cartographie. Bulletin des amis du musée préhistorique du Grand-Pressigny, Tours, 60 : 11-16.

LEA V., 2004. Les industries lithiques du Chasséen en Languedoc oriental : caractérisation par l'analyse technologique. British Archaelogical Reports International Series 1232, Oxford.

Mallet N., 1992. Le Grand-Pressigny : ses relations avec la civilisation Saône-Rhône. Bulletin des amis du musée préhistorique du Grand-Pressigny, numéro spécial.

Perrin T., 2003. Évolution du silex taillé dans le Néolithique haut rhodanien autour de la stratigraphie du Gardon (Ambérieu-enBugey, Ain). Presses Universitaires du Septentrion, Lille.

Ramseyer D., 1987. Delley-Portalban II. Contribution à l'étude du Néolithique en Suisse occidentale. Archéologie Fribourgeoise, 3, Fribourg.

Semenov S.A., 1964. Prehistoric technology. An experimental study of the oldest tools and artefacts from traces, manufacture and wears. Londres, éditions Cory, Adams and Mackay.

Tringham R., Cooper G., Odell G.H., Voytek B., Whitam A., 1974. Experimentation in the formation of edge damage : a new approach of lithic analysis. Journal of Field Archaeology, 1, 1-2:171-196.

VAN Gijn A. L., 1989. The wear and tear of flint, principles of functional analysis applied to dutch neolithic assemblages. Analecta praehistoria leidensia, 22, University of Leiden, Institute of prehistory.

Viellet A., 2009. Apport des études dendrochronologiques à la connaissance des sites lacustres de Chalain et Clairvaux (Jura). Clairvaux II-IIbis, Chalain 19 et Chalain 2, Gallia-Préhistoire, $51: 273-318$. 